\title{
Shallow water fisheries sonar: a personal view
}

\author{
Tim Mulligan* \\ Pacific Biological Station, Department of Fisheries and Oceans, Hammond Bay Road 3190, Nanaimo, BC V9R 5K6, Canada
}

This is the second international conference on the topic of fisheries sonar in shallow waters. One may ask, "Why have a conference on this specialised topic? What problems are characteristic of this field that necessitate a separate conference?" In these introductory comments to this conference, I shall mention several characteristics that may help focus attention on problems that are either unique to shallow water acoustics, or are more difficult to treat in this environment.

First, a definition of shallow water sonar will be helpful. Contrary to expectations, shallow water sonar need not be confined to shallow water. The critical feature is measurement of fish near a boundary. Thus, deep water sonar studies of fish near the bottom or near the surface share many of the characteristic problems of shallow water acoustics. The same will be true for fish near any natural or manmade boundary, such as a vertical precipice or a dam. The proximity to boundaries is the central factor. It is a much easier task to observe fish acoustically in mid water than it is near a boundary. Most of the effort in fisheries sonar over the past few decades has been spent on studies and surveys of mid-water fish. It is only relatively recently, with the advent of stable, sophisticated sonar equipment, that a growing effort has been placed on observations of fish near boundaries.

The sonar equipment typically used in most shallow water studies includes single-, dual- or split-beam echosounders. More recently, multi-beam, scanning and long range sonar systems have been used to help overcome some of the limitations imposed by the shallow environment. As the integration of software and hardware continues to increase, the spectrum of equipment useful for this type of work will no doubt broaden.

\section{VERY BRIEF HISTORY}

It is appropriate that this conference take place in Seattle, since much of the pioneering work on the use of sonar in rivers and lakes was done by people and organisations based in this city. Three of the manufac-

*Correspondence and reprints.

E-mail address: mulligant@pac.dfo-mpo.gc.ca (T. Mulligan). turers of modern split-beam echosounder systems have Seattle as their headquarters. The Applied Physics Lab of the University of Washington was also prominent in the early development and continues to contribute to the field. After the construction of dams on the Columbia River, resources were available to study the impact of these structures on the migration of salmon. These Seattle-based groups began their early riverine work at this time. Much of this early work focused on the migration of salmon smolts as they moved downstream towards salt water. Learning about the proportion of fish that passed through the turbine intakes on the dam and subsequent changes to these structures to reduce this proportion, provided the background for much of the early progress. Likewise studies of the behaviour and abundance of both juvenile and adult salmon in Lake Washington was the background for development of much of the equipment and methodology we use today.

Shallow water work has mirrored the mid-water work in its emphasis on estimation of fish abundance. Echo counting, trace counting and echo integration have all been applied to shallow water acoustic data. The prominence of the counting estimates is characteristic of shallow water studies because fish are

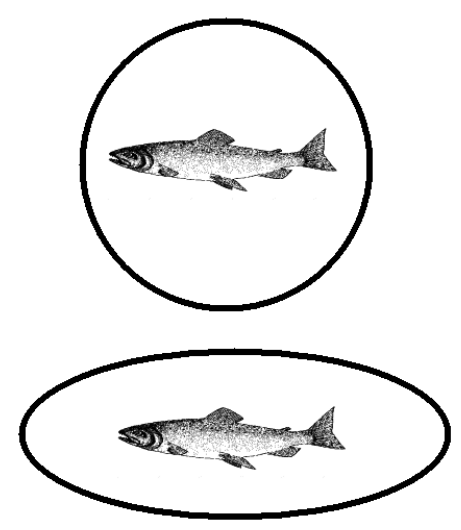

Figure 1. A diagram of fish size relative to beam size and shape for circular and elliptical beams. 


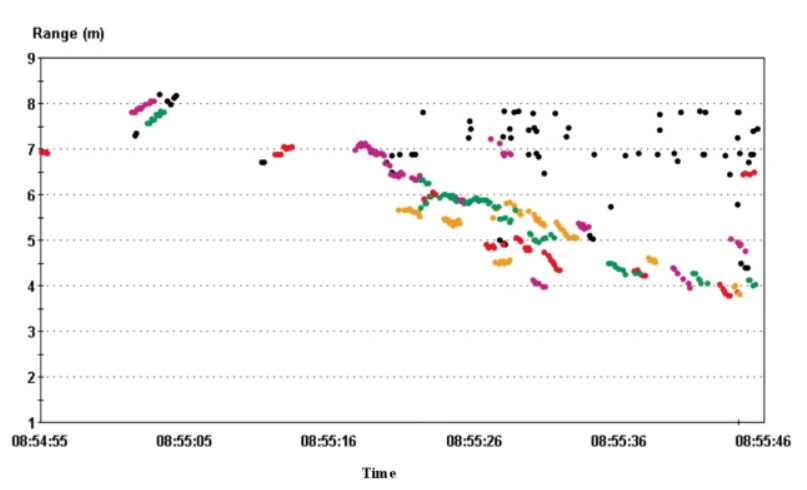

Figure 2. Fish at close range cause the appearance of intermittent targets from the boundary at greater range. Tracked fish are coloured, intermittent echoes are black.

typically observed at close ranges, so that echoes from individuals are resolved. Side-looking sonar is much more widely used in this field because of the advantages it offers in increased sampling volume. Looking parallel to a boundary, as opposed to perpendicular to it, is one way to address the near-boundary issue. But in addition to population estimates, shallow water work probably contains a larger proportion of studies on fish behaviour and migration than mid-water work. Consequently, methods to observe fish movement, such as fish tracking, have played a larger role.

More recently, a wider variety of problems have been addressed. The aquaculture industry has requirements for measuring the abundance and behaviour of fish in net enclosures. Acoustic measurements in this area also represent near-boundary applications. Here, fish densities are typically much higher than in the natural environment and secondary scattering and absorption can cause problems. Acoustic recognition of bottom types for habitat classification is also being tried. Recognising the appropriate bottom type for the species of interest has the potential to save substantial amounts of time when surveys are conducted.

(a)

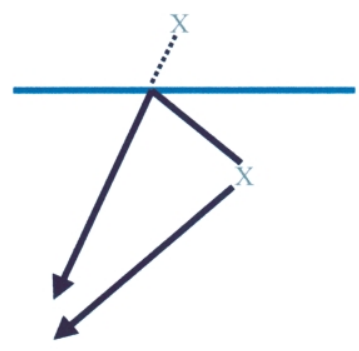

(b)
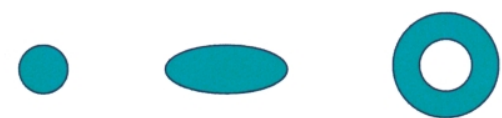

Figure 3. The Lloyd mirror effect. a) Beams from a target near the surface and its reflected image interfere. b) Interference patterns produced for a point target subject to this effect.

\section{CHARACTERISTICS AND PROBLEMS ASSOCIATED WITH SHALLOW WATER SONAR}

Detection of fish near a boundary is difficult because of the potential for interference with, or distortion of, the fish signal. The classical condition for fish detection required either that the fish be at a lesser range than the boundary, or that the fish signal be greater than that from the boundary. Often times, these conditions are not easily achievable in shallow water. Horizontal-looking sonar beams can help in such cases. Looking parallel to a boundary is one method of increasing the detection probability for fish located near the boundary. This method will help in cases where the boundary and the fish are observed at the same range by reducing the amplitude of the boundary echo due to the oblique incidence of the beam. Other methods can be tried that exploit some special properties of the fish and the boundary. For example, in the case of a fixed boundary and moving fish, subtracting the mean signal versus range from each incoming ping may allow one to observe the fish in the presence of a highly reflective boundary. One needs to be careful with this technique, since the background signal will be variable and therefore the observed fish signal will be affected.

The distribution of fish in the water column is typically not uniform. Fish are often attracted to boundaries for shelter, food, nesting sites, reduced current flow and a variety of other reasons. This results in a higher density of fish near the boundary, where they are more difficult to observe. In this way, as in many other aspects of life, nature is not random, it is perverse.

Horizontal looking sonar has some undesirable consequences. One of these is that the fish's aspect angle with respect to the beam will usually be more variable than with conventional downward-looking sonar. Fish behaviour is such that dorsal or ventral aspect is more

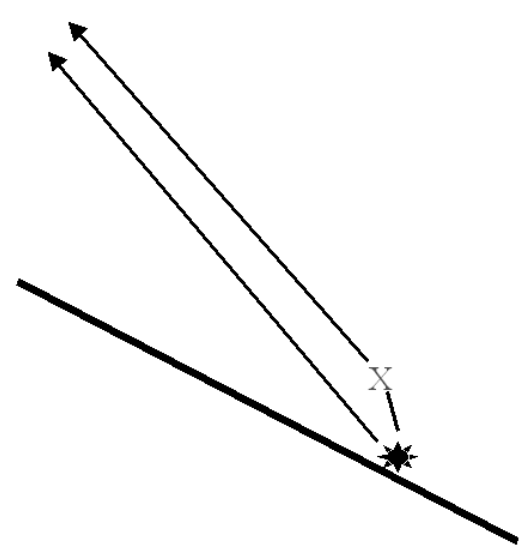

Figure 4. Sound that is forward-scattered from a target is subsequently scattered from an object on the boundary. 


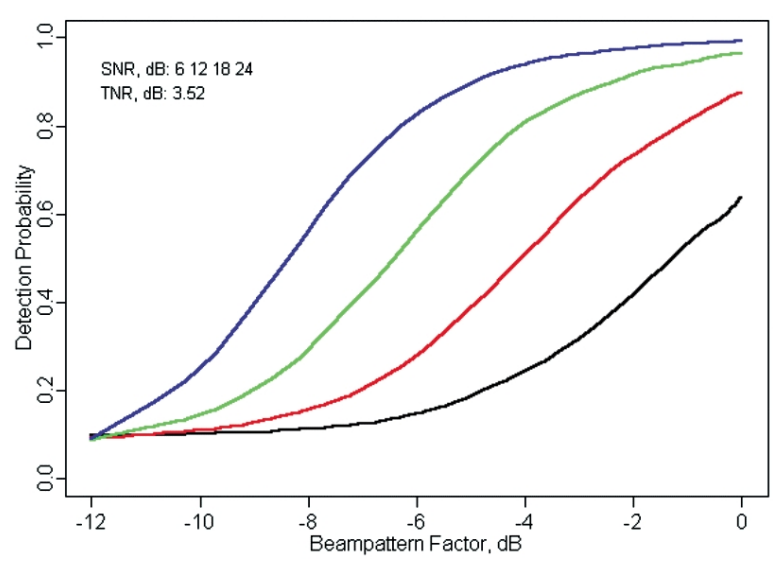

Figure 5. Fish detection probability vs. beam pattern factor for several signal-to-noise ratios.

consistent than side aspect. High variability in aspect angle results in high variability in detection probability. Both counting and integration will be severely degraded by this effect. In situations where there is a current flow, aspect angle may be less variable due to the alignment of the fish with the current. Transducer placement can then aid in increasing fish detection by aiming perpendicular to the current flow.

Horizontal looking sonars must be more concerned with other factors that are typically negligible with downward looking systems. One of these factors is the influence of gradients in water characteristics (such as salinity or temperature) on the propagation of sound. Since gradients are typically in the vertical direction, downward looking sonar are normally not affected by them. By contrast, horizontal looking sonar will be affected and can experience curved, rather than straight-line sound propagation. For long ranges and large gradients, this represents a serious problem. Another factor is the increased importance of the spatial orientation of the beam with respect to the boundary. Since the goal is to observe close to the boundary, knowing its shape and the positioning of the beam along the boundary is crucial to successful sampling.

Irrespective of the aiming of the sonar beam, in shallow water environments we often observe fish near the transducer. If the fish is in the near field of the transducer, not much useful information can be obtained. However, this can also be troublesome for cases where the fish is beyond the near field. Part of the problem is that a fish at close range is not a point-source target (figure 1). Thus, the measurement of its target strength and three-dimensional position will be affected. This can be partially compensated by using an elliptical beam cross section oriented to conform with the fish's orientation. A second feature with short range fish detection is that the fish itself disrupts a substantial portion of the beam. The situation is similar to passing a lens in front of a flashlight beam. The geometry of the light beam beyond the lens

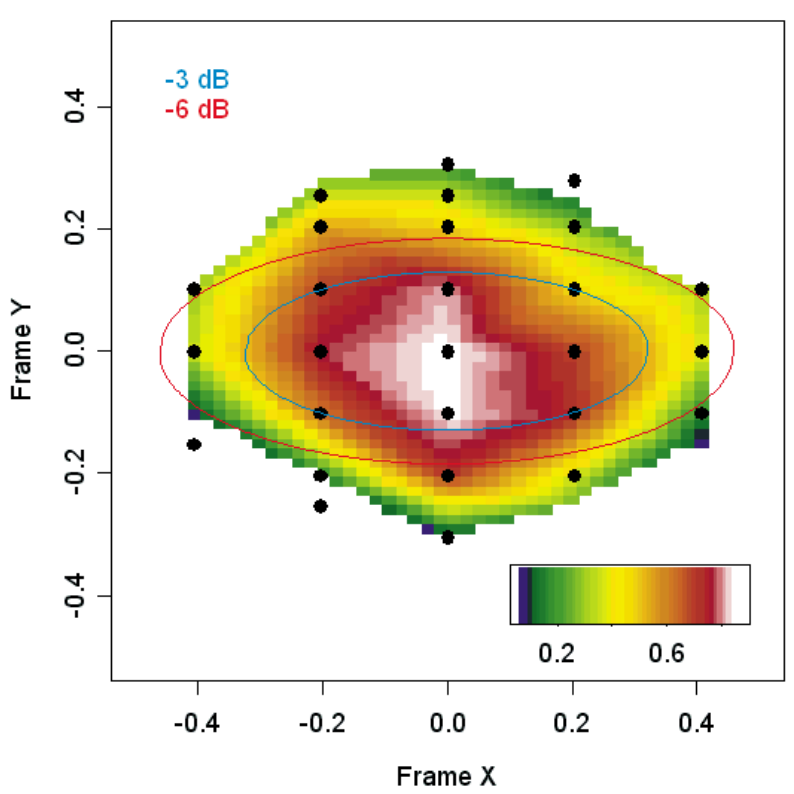

Figure 6. A map of fish detection probability measured with a salmon target and a $4^{\circ} \times 10^{\circ}$ elliptical transducer. Beam contours at -3 and $-6 \mathrm{~dB}$ are shown for reference. The black dots show the locations at which the measurements were made.

(and of the sound beam beyond the target) will be affected. The result is that intermittent echoes from the boundary are often correlated with fish targets close to the transducer (figure 2).

Boundaries can also be sources of interference from primary and secondary scattering. Both the surface and the bottom cause primary scattering due to specular reflection from their uneven surfaces. The water adjacent to the surface is often the source of volume reverberation due to entrained air bubbles generated by wave action or precipitation. A mirror-like surface, in addition to reflecting the incident beam, can generate secondary scattering interference between a shallow target and its mirror image (figure 3). This effect (similar to the Lloyd mirror effect in the field of optics) will disrupt the amplitude and phase of the returning echo so that measurements of the target's size and position will be much more variable and erratic. Another example of secondary scattering occurs when there is forward scattering from the fish that is then backscattered from an object at greater range (figure 4 ). This is the mechanism responsible for the intermittent targets shown in figure 2.

Moving water imparts characteristic problems for fisheries acoustics. In the rivers where I monitor migrating adult salmon, we are faced with a low signal-to-noise ratio. Typically this ratio will be in the range of $10-15 \mathrm{~dB}$ and is caused by high background noise due to volume backscattering (reverberation) from bubbles, turbidity and debris in the water. We use a split-beam echosounder. The major problem that results from a low signal-to-noise ratio is lowered fish 


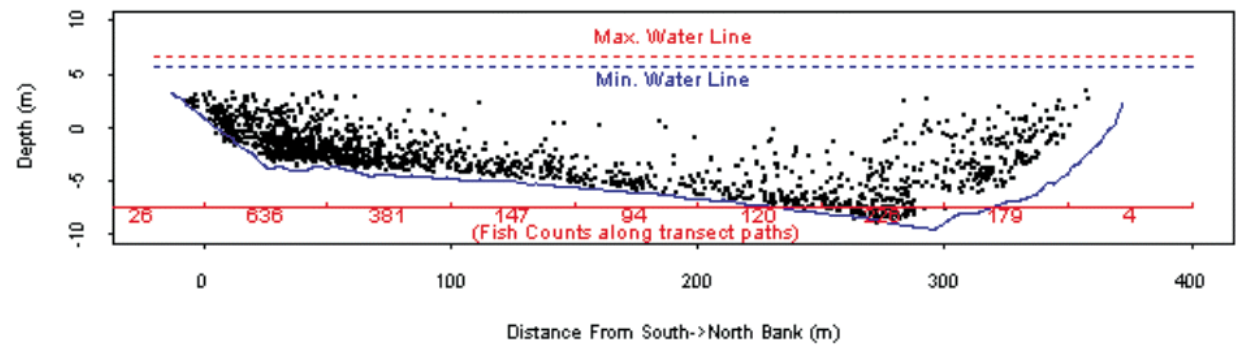

Figure 7. Cross sectional view of the Fraser River with tracked fish positions shown by dots. Fish prefer to migrate near shore and near the bottom where current is low. detection probability (figure 5). This is particularly frustrating for horizontal beams aimed close to the bottom. Fish migrating close to the bottom will pass through the lower portion of the beam for which detection probability is low (figure 6). Upstream migrating fish routinely swim close to the banks and the bottom where the current is lower (figure 7). This exacerbates the problem of fish detection.

The decreasing current near the bottom often causes a gradient in fish density in the water column, with higher density in the regions of lower current. For a side-looking sonar beam, this can result in nonuniform fish density over the beam cross section. All three of the standard fish density estimation techniques, echo counting, trace counting and echo integration, assume uniform density over the beam cross section. Therefore, these techniques may have to be modified to avoid biased estimates.

The final problem associated with moving water that I shall mention is the potential for a dynamic boundary. One particularly insidious example of this phenomenon is that of sand waves that migrate downstream along the bottom of some rivers. These waves are analogous to moving sand dunes in deserts. All of the problems of measuring fish near boundaries are increased when the boundary itself is not constant.

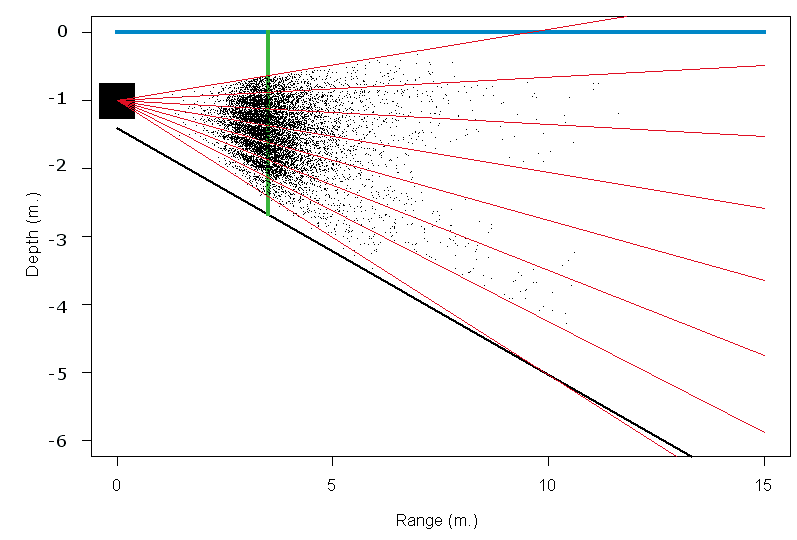

Figure 8. A plot of fish distribution with respect to real world co-ordinates. Data from 7 separate aiming angles of the transducer are shown.

\section{PERSONAL THOUGHTS}

We all think about acoustics from our own, unique perspective. One prerogative of being asked to give this talk is the opportunity to inject some of my personal thoughts on the subject and I shall now subject you to them. First, fisheries sonar is an excellent example of an interdisciplinary field of research. An ideal research team should include members from several disciplines. Physics and engineering provide the theoretical foundation for sound propagation and scattering. The development of new measurement techniques and equipment rely heavily on these areas of knowledge. Mathematics and statistics play a vital role in data analysis, sampling theory and design, simulation, and modelling. Biology is important when working with living organisms. This field is essential to the understanding of fish behaviour, habitat preferences, food requirements and many other issues relevant to when, where and how to find fish. Controlling undesirable fish behaviour may be an option in some cases. If the problem has a fisheries management component, this field should also be represented. Finally, computer science is the arena in which data storage, equipment control, data analysis, modelling and simulation all converge.

Experience has taught me that sonar data are highly subject to artefacts. I have mentioned several of them in this talk. Sonar is not WYSIWYG (an acronym for "what you see is what you get"). To be able to overcome the distortions that are inherent in our observations, we need to understand the measurement system. Whenever we can identify and explain an artefact, we have the potential to remove or compensate for it.

My tendency is to compartmentalise fisheries sonar, i.e., to think of it as strictly an area in which the field of acoustics provides the sole tool. However, there is great potential for combining sonar with other types of measurements. The best idea I have learned about recently involves the combination of sonar and a resistivity counter. These counters operate by measuring the perturbation in an electric field as a fish swims past. One problem with this type of device is that the fish must swim very close to the field-generating electrodes to be detected. Thus, they measure fish well when the range is small. If such a counter were placed 
Figure 9. The top panel shows the scatter plot of fish swimming speed versus depth. The bottom plot shows speed versus depth and distance from shore.
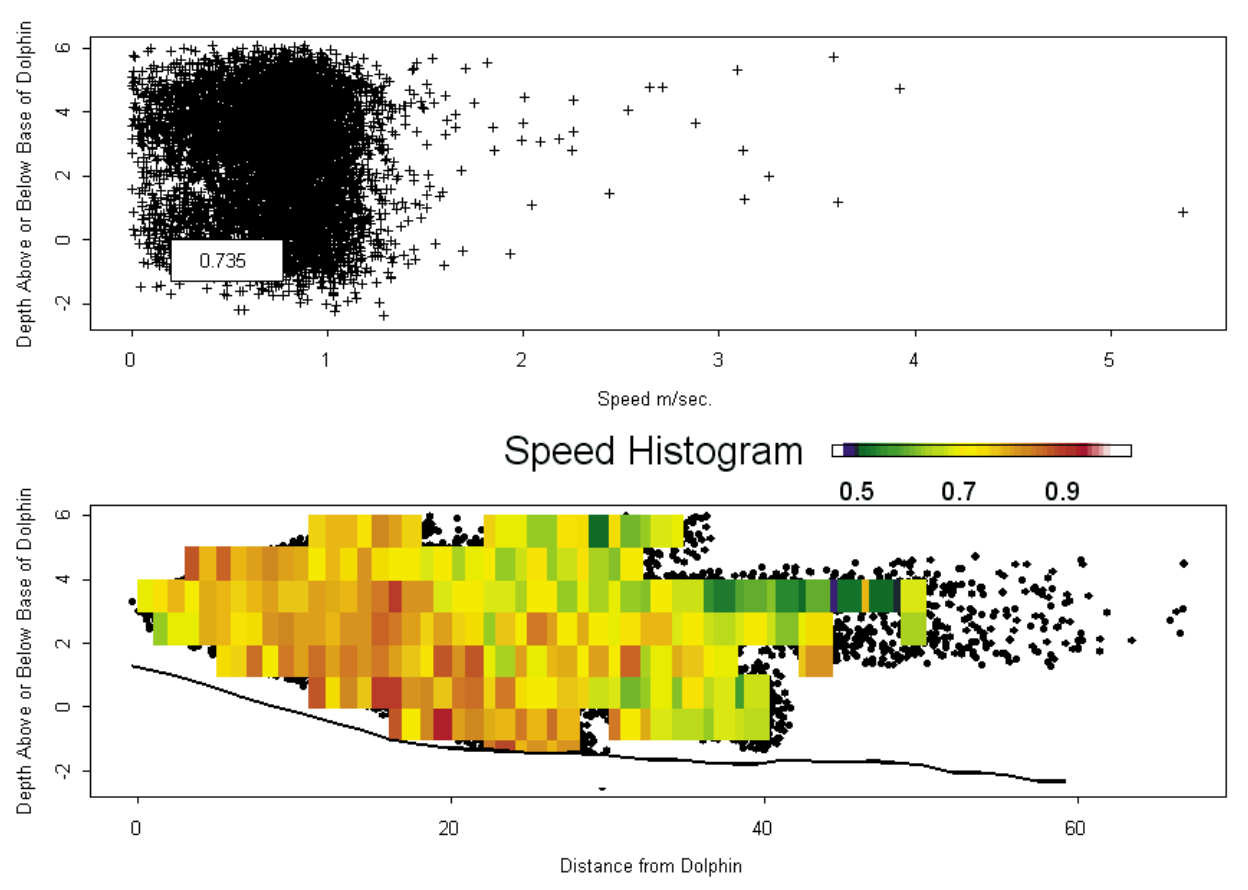

on the boundary, it might provide the ideal complimentary measurement to sonar data.

Finally, there is a tendency for us as sonar people to leave the data in sonar-related form. We typically look at echograms, target strength distributions and so on. We can often benefit by putting our observations back into the real world. This helps us get an overview and see things from the fish's perspective. As an example, plots of fish distributions with respect to real world co-ordinates, as opposed to within-beam co-ordinates, can be quite informative (figure 8). Seven separate aiming angles were used to collect these data. The data from any individual aiming angle contains a fraction of the information on the spatial distribution of the fish. The figure also shows a systematic pattern of the fish distribution that crosses the boundaries between the aiming angles. The fact that this pattern does not appear to be discontinuous at the junctions between the separate aims, gives us confidence that we are observing something real and related to the fish's spatial distribution, rather than some artefact of the measurement process. A final example shows consistency of the data that is apparent only when the data are examined in two dimensions (figure 9). The top plot indicates that a fish's swimming speed is unrelated to its depth. The bottom plot uses a coloured matrix to display a two-dimensional histogram of swimming speed versus depth and distance from shore. This histogram overlays a plot of the fish spatial distribution shown by the black dots. When the number of fish observed within a $1 \mathrm{~m} \times 1 \mathrm{~m}$ cell is greater than 20 , the cell in the 2-D histogram is coloured to show the mean speed of these fish. The 2-D histogram shows that there is a pattern in the spatial distribution of swimming speed. Fish near the bottom and near the bank generally swim faster. This is to be expected, since the current flow is lower in these same areas. The point of the figure is that real world entities, such as fish, often respond to multidimensional factors. If we confine ourselves to looking for systematic relationships one variable at a time, we may miss the message.

To conclude this introduction, I would like to encourage each of you not to let the equipment and technicalities of our work obstruct the underlying beauty and satisfaction of studying and working with nature. I hope you can take time to enjoy the aesthetics of your work. Our ultimate goal is not to subjugate nature, but to be responsible stewards. If we are successful, humans may eventually learn to live within nature, rather than as a separate, dominant entity. 